\title{
Brain Tumor Detection Using Wathershed Segmentation Techniques and Area Calculation
}

\author{
Meenakshi Pareek*, C.K Jha, Saurabh Mukherjee, Chandani Joshi \\ Department of Computer Science Banasthali University, Rajasthan, India
}

\begin{tabular}{l}
\hline \hline Article Info \\
\hline Article history: \\
Received Apr 8, 2018 \\
Revised Jun 19, 2018 \\
Accepted Jul 02, 2018 \\
\hline
\end{tabular}

\section{Keyword:}

Gardient magnitude

MRI

Sobel

Watershed segmentation

\begin{abstract}
This paper primarily focuses on to employ a novel approach to classify the brain tumor and its area. The Tumor is an uncontrolled enlargement of tissues in any portion of the human body. Tumors are of several types and have some different characteristics. According to their characteristics some of them are avoidable and some are unavoidable. Brain tumor is serious and life threatening issues now days, because of today's hectic lifestyle. Medical imaging play important role to diagnose brain tumor .In this study an automated system has been proposed to detect and calculate the area of tumor. For proposed system the experiment carried out with $150 \mathrm{~T} 1$ weighted MRI images. The edge based segmentation, watershed segmentation has applied for tumor, and watershed segmentation has used to extract abnormal cells from the normal cells to get the tumor identification of involved and noninvolved areas so that the radiologist differentiate the affected area. The experiment result shows tumor extraction and area of tumor find the weather it is benign and malignant.
\end{abstract}

Copyright (C) 2018 Institute of Advanced Engineering and Science. All rights reserved.

\section{Corresponding Author:}

Meenakshi Pareek,

Department of Computer Science Banasthali University,

Rajasthan, India.

Email: pmeenakshi86@gmail.com

\section{INTRODUCTION}

Abnormal development of tissues in the brain causes the brain tumor. Basically BRAIN is the core part, in the human body, every part have different cells and all cells have their own capabilities, some cell grows with their own functionality and some lose their capability and resist and grow aberrant. These mass collections of the cells form the tissue which is called as "tumor". Among those cells some are cancerous or some are non-cancerous. Based on those cells, the tumor can defined in two categories benign and malignant. Central Brain Tumor Registry of the United States (CBTRUS) in December 2015 published a report, according to this statistical report brain tumor is the second leading cause of death in children and Leukemia is first [1]. Basically brain tumor categorized into two parts: Benign and Malignant. The World Health Organization (WHO) had classified tumor into 4 Grades, Grade I to IV. Grade I and grade II are low grade which represents Benign Tumor. Grade III and Grade IV are high grade which represents malignant tumor [2]. Many different imaging modalities are used in the last two decades to identify and locate the anatomical structure such as XRay, CT scans and MRI [3]. But due to the change in time the high quality images are used to determine tumor, which is known as MRI (Magnetic Resonance Image). T1 weighted and T2 weighted images are part of the MRI [4]. Anatomic behavior of brain is shown through the MRI. MRI is also used for the study the internal structure of the human body. Radiologist visualizes and analyzes those images. MRI techniques completely based on magnetic field [5]. In MRI electromagnetic waves are used and these waves are transmitted in the human brain and signal are recorded and reconstructed on images and these images are analyzed by computer program. Shown in Figure 1. 


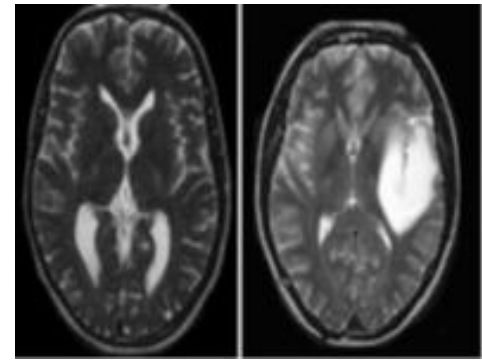

(a) (b)

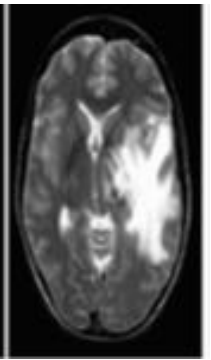

(c)

Figure 1. (a) Normal Brain, (b) Benign Tumor, (c) Malignant Tumor

In medical image processing, segmentaion plays an important role for modeling of brain and construct brain atlases. It's a challenging task for us to segment proper region because DICOM image have a variety of information, such are location, shape, intensity, etc. So, that it is a tedious task to segment region of interest. For segmentation, medical imaging has different segmentation approaches, like edge based, threshold base, region growing etc [6].

\section{SEGMENTATION}

To carry out image segmentation, there are many existing segmentation techniques. Segmentation plays key task for Medical images to perform pre and post surgical planning, early detection. To carry out image segmentation, there are many existing segmentation techniques. Segmentation plays key task for medical images to perform pre and post surgical planning, early detection.

\subsection{Region Based Segmentation}

Region based segmentation technique include region growing and region splitting and merging. By region growing method, pixels are grouped in a sub region from large region. In this approach one "seed" point find out firstly, where the neighboring pixels are growing, that have identical characteristics of the seed [7]. In region splitting method, growing regions are split in top down direction, in splitting remote part is more homogeneous than the whole. If adjacent region are get, selected regions are merged using region merging method in which week boundaries are merged and robust edges are embossed. In region growing split and merge method is the ambiguous. Every region could be divided into sub regions, and the suitable regions could be merged into a one region [8].

\subsection{Thresholding Based Segmentation}

Thresholding is another technique of segmentation. It is an intensity based method of segmentation. In this method firstly find the pixel value of images, so that the region of interest segment. Then apply threshold value has applied to image pixel values, those regions have the black background and higher intensity can also be segmented [9]. Threshold value is decided on the basis of adjacent pixel, and get segmented part [10]. Because of intensity dependent image have noise and weak boundaries, to remove noise detect boundaries some morphological operations are used with threshold methods [7].

\subsection{Edge or Boundary Based Segmentation}

Because of the intensity some edges are strong and some of them are strong. Image edges are depending on intensity of pixels. Some lines are roof and ramp edges. If the in the image gray tone is high, then it transforms in the edges. The edges represent continuity and discontinuity of an object. To detect edges perwitt edge detection, robert edge detection and sobel edge detection methods are used for edge detection [11].

\section{PROPOSED METHODOLOGY}

The proposed system mainly has four modules, Image acquisition, segmentation, tumor detection and area calculation and coverage of the tumor. Segmentation is carried out with watershed technique. The following Figure 2 showing the proposed work. 


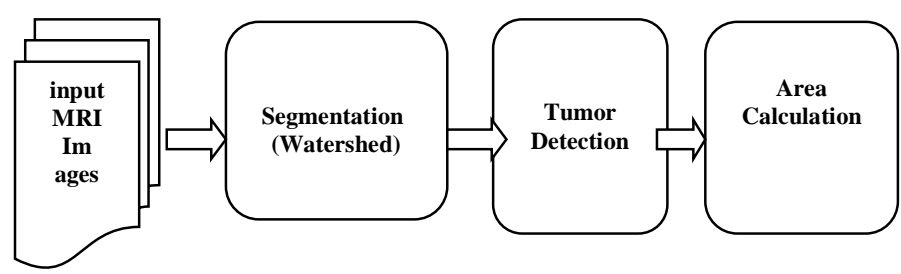

Figure 2. Process of Brain Tumor Segmentation and Its Area Calculation

In this section, we proposed a system that could segment and extract the tumor from MRI images. All experimental work is done with MRI images and MATLAB. The following steps are used to detect and segment the tumor and find its area.

a. Input MRI image.

b. Convert image in gray image using rgb2gray function in MATLAB for segmentation.

c. In the third step remove all small connect object those are have fewer than pixel $\mathrm{P}$ from binary image using a bwareaopen function in MATLAB.

d. In the fourth step suppress light structures connected to image border with imclearborder function.

e. In the fifth step, for gradient of the image using Sobel Operator.

f. In the sixth step watershed is applied to extract different pixels from the background for segmentation and detection.

g. At the last step calculate the area of the tumor segmented part

\subsection{Image Acquisiition}

The proposed experiment carried out with different module. Image acquisition is first module. We had done experimentation with $150 \mathrm{MRI}$ images taken from "figshare brain data set". This brain tumor dataset containing 3064 T1-weighted contrast-enhanced image of 233 patients with two kinds of brain tumor, for the experiment $50 \mathrm{MRI}$ images are carried out.

\subsection{Watershed Segmentation}

There are many segmentation techniques. In our work we have used watershed. Watershed segmentation is a famous "edge based segmentation" algorithm. Basically watershed term is used in geography also, related to the water, means water is drained in the particular area [3]. The watershed segmentation depends upon the flow of water, when two different water bodies are meeting then they build dams. The water mounted until all peaks in the map are absorbed. In image processing the dams are "Watershed" and images are segmented by dams and all segmented part is catchment basin [12]. Mainly watershed is "Gradient Based Segmentation" method, gradient values depend on the pixel's intensity. The higher intensity gradient region is "Watershed", which is divided in nearest local minima like "Basin" [13].

In this paper watershed segmentation have used for brain tumor segmentation from MRI images. We assume $\mathrm{h}$ is highest intensity point, for the image gradient mapping, we check all the pixels in $\mathrm{h}$, and segment image using their nearest pixel [14]. Following steps are for watershed segmentation technique, how it will work.

a. We check the neighboring pixel from catchment then, we examine the neighboring pixel in height $h$, if we found the pixels then we labeled it and repeat this process.

b. If our searched pixel is in nearest pixel and have two different Labels then we declare this as a new Label W that is our "watershed pixel". In this situation a dam is built which separate 2 basins or 2 different Labels.

c. After that, all watershed pixels are club and segment the object which we want to segment. At the final step where we get what we find means there is no more neighboring pixel to check in $\mathrm{h}$ [14].

Shown in Figure 3 and Figure 4. 


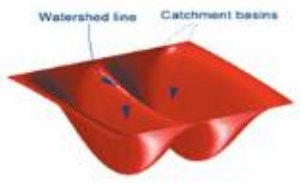

Figure 3. Watershed Segmentation with Catchment Basin

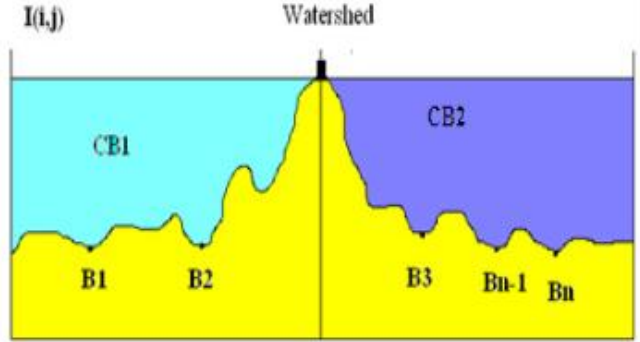

Figure 4. Watershed Principle of Simplified To Two Dimension

Watershed segmentation is edge based segmentation which first uses image acquisition technique and then the gradient magnitude of the image is performed [15]. Gradient of $f$ at coordinates ( $x, y)$ is defined as two dimensional column vector shown in Equation (1) and the magnitude of vector $\nabla \mathrm{f}$ denoted as $\mathrm{M}(\mathrm{x}, \mathrm{y})$ as in Equation (2). Gx And gx are sobel operators, called as mask coefficient 3, shown in Equation (3) and (4) [7]. In sobel operator two convolution kernel are used vertical and horizontal to detect contrast [16].

$$
\begin{aligned}
& f=\operatorname{grad}(f)=\left[\begin{array}{l}
g x \\
g y
\end{array}\right]=\frac{\frac{\partial f}{\partial x}}{\frac{\partial f}{\partial y}} \\
& M[x, y]=\operatorname{mag}(\nabla f)=\sqrt{g x^{2}+g y^{2}} \\
& g x=\frac{\partial f}{\partial x}=\left(Z_{7}+2 Z_{8}+Z_{9}\right)-\left(Z_{1}+2 Z_{2}+Z_{9}\right) \\
& g y=\frac{\partial f}{\partial x}=\left(Z_{7}+2 Z_{8}+Z_{9}\right)-\left(Z_{1}+2 Z_{2}+Z_{9}\right)
\end{aligned}
$$

\section{RESULT AND DISCUSSION}

In this section we discuss about the experiment result. For segmentation and to detect tumor we developed GUI in MATLAB. The tumor is segmented, using the watershed segmentation method. Watershed segment is very useful in medical field because this method solve the pixel overlapping problem in gray scale image.

We developed a GUI for our proposed system, firstly system take MRI images from database, and extract segmented tumor part. With the help of thresholding, we decide whether the tumor is "Benign" and "Malignant" and calculated the tumor coverage and tumor area. We calculate the tumor area on three different cases of images such as non tumor, benign tumor and malignant tumor. The following GUI shows the three different cases. In case of normal brain image we get the tumor area is $0 \mathrm{~mm}^{2}$ and no segmented tumor part.

\section{BINARY ROBUST INVARIANT SCALABLE KEY POINTS (BRISK) THE METHOD}

Description of the key stages in BRISK, namely feature detection, descriptor composition and key point matching to the level of detail that the motivated reader can understand and reproduce. It is important to note that the modularity of the method allows the use of the BRISK detector in combination with any other key point descriptor and vice versa, optimizing for the desired performance and the task at hand [9]. Shown as in Figure 5, Figure 6, and Figure 7. 


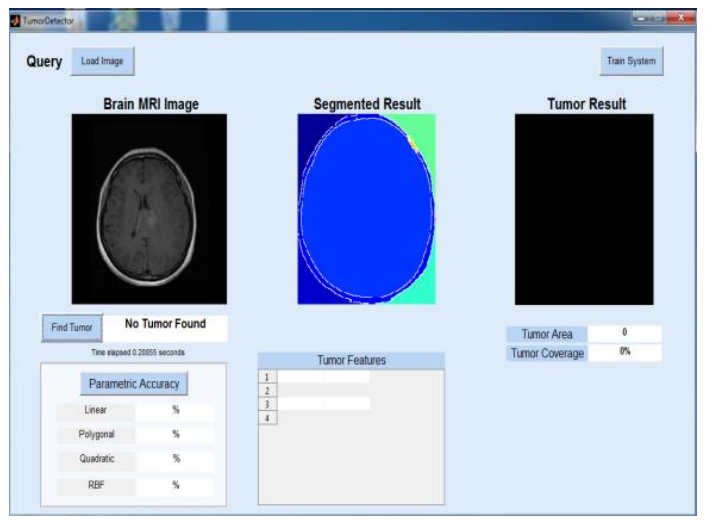

Figure 5. GUI for Normal Brain Image

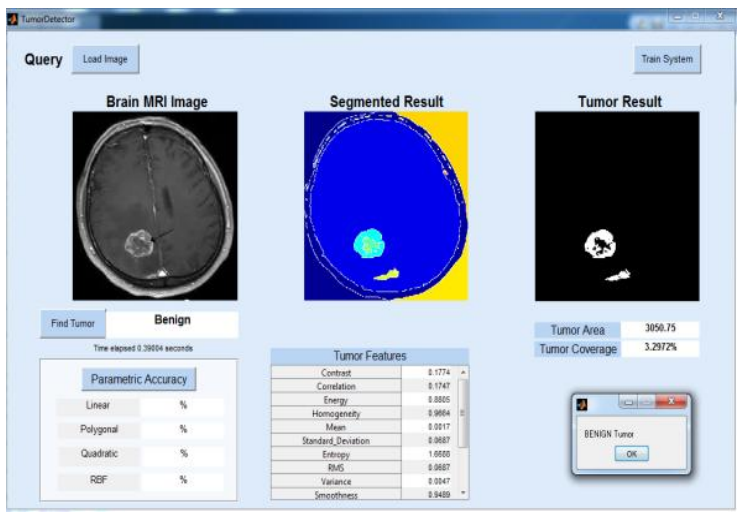

Figure 6. GUI for Benign Tumor Image

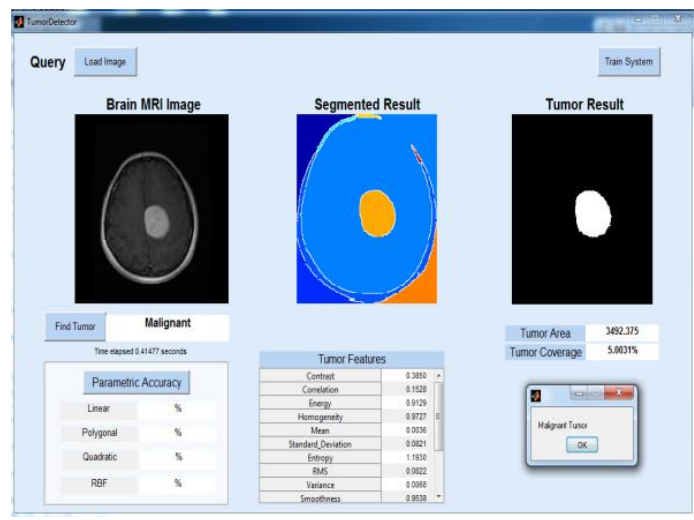

Figure 7. GUI for Malignat Tumor Image

\section{CONCLUSION AND FUTURE WORK}

In medical image processing there are so many segmentation techniques. In this paper we present an automated system to detect and classify the brain tumor. Thesholding is applied to extract object from background. For the segmentation we used watershed method and we classify the tumor in two classes "Benign" and "Malignant". We calculate the tumor area with three cases such as normal brain image, malignant image and benign image. We get $0 \mathrm{~mm}^{2}$ with normal image.

Watershed segment have one disadvantage is over segmentation. To overcome this advantage marker controller are used. In our future work we use watershed method with marker controller and some fuzzy techniques and classify the tumor with SVM classifier.

\section{REFERENCES}

[1] Sehgal, A., Goel, S., Mangipudi, P., Mehra, A., Tyagi, D., "Automatic brain tumor segmentation and extraction in MR images. In Advances in Signal Processing (CASP)", Conference on (pp. 104-107), June 2016. IEEE.

[2] Liu, J., Li, M., Wang, J., Wu, F., Liu, T., Pan, Y., "A survey of MRI-based brain tumor segmentation methods". Tsinghua Science and Technology, 19(6), 578-595. 2014.

[3] Dhage, P., Phegade, M. R., Shah, S. K., "Watershed segmentation brain tumor detection. In Pervasive Computing (ICPC)", 2015 International Conference on (pp. 1-5), January 2015. IEEE.

[4] Sharma, M., \& Singh, S., "A Modified and Improved Method for Detection of Tumor in Brain Cancer". International Journal of Computer Applications, 91(6), 2014.

[5] Gondal, A. H., \& Khan, M. N. A., "A review of fully automated techniques for brain tumor detection from MR images". International Journal of Modern Education and Computer Science (IJMECS), 5(2), 55.

[6] Bawage ,R. chandane, N., Phad V. Zargar, S., "K-Means Clustering based Brain Tumor Detection and Area Calculation in MRI With Graphical user Interface". International Journal of Engineering Research \& Technology (IJERT), 4(11): 378.

[7] Baraiya, N., \& Modi, H., "Comparative study of different methods for brain tumor extraction from MRI images using image processing". Indian Journal of Science and Technology, 9(4), 2016.

[8] Sonawane, M. S., Dhawale, C. A., "A brief survey on image segmentation methods". In IJCA Proceedings on National conference on Digital Image and Signal Processing. 2015.

Brain Tumor Detection Using Wathershed Segmentation Techniques and Area ... (Meenakshi Pareek) 
[9] Lakshmi, A., Arivoli, T., "Brain Tumor Segmentation and its Area Calculation in Brain MR Images using K-Mean Clustering and Fuzzy C-Mean Algorithm". 2015.

[10] Khan, M. W., "A survey: image segmentation techniques". International Journal of Future Computer and Communication, 3(2), 89, 2014.

[11] Zaitoun, N. M., \& Aqel, M. J., "Survey on image segmentation techniques”. Procedia Computer Science, 65, 797-806, 2015.

[12] Balafar, M. A., Ramli, A. R., Saripan, M. I., Mashohor, S., "Review of brain MRI image segmentation methods". Artificial Intelligence Review, 33(3), 261-274, 2010

[13] Salman, N., "Image segmentation based on watershed and edge detection techniques". Int. Arab J. Inf. Technol., 3(2), 104-110, 2006.

[14] Jung, C. R., Scharcanski, J., "Robust watershed segmentation using wavelets". Image and Vision Computing. 23(7), 661-669, 2005

[15] Salman, S. D., \& Bahrani, A. A., "Segmentation of tumor tissue in gray medical images using watershed transformation method", International Journal of Advancements in Computing Technology, 2(4), 2010

[16] Joshi, C., Purohit, G. N., Mukherjee, S., Multispectral Satellite Image Retrieval Using The Combination of Features Color, Shape and Texture. 